DAMTP/96-18

gr-qc/9604012

\title{
Consistent Sets Yield Contrary Inferences in Quantum Theory
}

\author{
AdRIAN Kent园 \\ Department of Applied Mathematics and Theoretical Physics, \\ University of Cambridge, \\ Silver Street, Cambridge CB3 9EW, U.K.
}

\begin{abstract}
In the consistent histories formulation of quantum theory, the probabilistic predictions and retrodictions made from observed data depend on the choice of a consistent set. We show that this freedom allows the formalism to retrodict contrary propositions which correspond to orthogonal commuting projections and which each have probability one. We also show that the formalism makes contrary probability one predictions when applied to Gell-Mann and Hartle's generalised time-neutral quantum mechanics.

PACS numbers: 03.65.Bz, 98.80.H
\end{abstract}

To appear in Phys. Rev. Lett.

1 Email: apak@damtp.cam.ac.uk 


\section{Introduction}

The consistent histories approach to quantum theory, pioneered by Griffiths [1,2], Omnès [3,4], and Gell-Mann and Hartle [5, 6, ,7], is perhaps the best attempt to date at a precise formulation of quantum theory that involves no "hidden" auxiliary variables and can be applied to closed systems. Since modern ideas in cosmology and quantum gravity require an interpretation of the quantum theory of the universe, the approach has naturally attracted a good deal of interest. It seems, however, that without new axioms, whose precise form is presently unknown, it is impossible to derive the standard predictions of classical mechanics and Copenhagen mechanics from the consistent histories formalism, since predictions essentially always depend not only on known data and the hamiltonian but on the selection of a particular consistent set [8]. This is true even if predictions are made conditional on the persistence of quasiclassical physics [9]. It should be said, nonetheless, that debate over the scientific status of the formalism continues and that its proponents tend to regard its lack of predictive power with more equanimity than do its critics.

This letter, though, looks at the logical properties of the consistent histories formalism rather than interpretational questions. It is helpful immediately to introduce a little logical terminology. We say that two projection operators $P$ and $Q$ are complementary if they fail to commute: $P Q \neq Q P$. We say that they are contradictory if they sum to the identity, so that $P=1-Q$ and $P Q=Q P=0$. Finally, we say that they are contrary if they are orthogonal and not contradictory, so that $P<1-Q$ and again $P Q=Q P=0$.

The somewhat counterintuitive properties of consistent sets of histories have, of course, already been extensively investigated in the original literature [1, 3, 5] and elsewhere [8,10]. We describe here a feature which seems to have gone unnoticed, namely that different consistent sets extending a given history can imply, with probability one, propositions which are contrary.

It is well known that the predictions and retrodictions made in different sets generally correspond to complementary projections and so are incompatible. This, in itself, might be taken as merely an extension to the consistent histories context of the familiar fact that non-commuting observables cannot simultaneously be assigned values. The inferences we consider here, though, correspond to commuting but orthogonal projections. The fact that they are nonetheless each assigned probability one in their respective sets is a result with no parallel in standard quantum theory. It raises the question of whether the present version of the consistent histories formalism is a natural generalisation of Copenhagen quantum mechanics. 


\section{The Consistent Histories Formalism}

We begin with a brief description of the simplest version of the consistent histories formulation of non-relativistic quantum mechanics, in which sets of histories correspond to sets of projective decompositions. While consistent histories can be defined abstractly on any Hilbert space $\mathcal{H}$, it is generally assumed that operators corresponding to the hamiltonian $H$ and other physically interesting observables, such as position, momentum and spin, are given. The dynamics, however, are irrelevant to the examples we consider, so that we will take $H=0$ and will not need to distinguish any particular operators as simple physical observables.

We are interested in a closed system whose initial density matrix $\rho$ is given. We will also be interested in applying the formalism to a version of the time-neutral generalisation of quantum mechanics first discussed by Aharonov, Bergmann and Lebowitz[11]. This can be thought of as a non-relativistic version of the theory which would be obtained by imposing initial and final conditions in quantum cosmology, and requires an initial density matrix, which we take to have the standard normalisation and write as $\rho_{i}$, and final boundary conditions defined by a positive semi-definite matrix $\rho_{f}$ normalised so that $\operatorname{Tr}\left(\rho_{i} \rho_{f}\right)=1$. The initial and final matrices $\rho_{i}$ and $\rho_{f}$ then give boundary conditions for the system at times $t_{i}$ and $t_{f}$, with $t_{i}<t_{f}$.

It may be helpful to think of the system under discussion as a non-self-interacting quantum system that is prepared in the state $\rho_{i}$ at $t=t_{i}$ and then isolated until $t=t_{f}$, when it is observed in the state $\rho_{f}$. The Hilbert space $\mathcal{H}$ here should then be thought of as a subspace of the full Hilbert space, which includes the degrees of freedom of the preparation and measuring apparatuses, and the full Hamiltonian is nonzero. In the examples we consider, both $\rho_{i}$ and $\rho_{f}$ are pure and $\mathcal{H}$ is three-dimensional or higher. Such examples can, of course, easily be realised in the laboratory.

The physical propositions we are interested in correspond to members of sets $\sigma$ of orthogonal hermitian projections $P^{i}$, with

$$
\sum_{i} P^{i}=1 \quad \text { and } \quad P^{i} P^{j}=\delta_{i j} P^{i}
$$

These projective decompositions of the identity should be thought of as being applied at definite times. The times are usually appended to the sets of projections, so that $\sigma_{j}\left(t_{j}\right)=$ $\left\{P_{j}^{i}\left(t_{j}\right) ; i=1,2, \ldots, n_{j}\right\}$ defines a set of projections obeying (2.1) and applied at time $t_{j}$. However, as our results depend only on the time ordering, we will omit explicit time labels 
and take sets of the form $\mathcal{S}=\left\{\sigma_{1}, \ldots, \sigma_{n}\right\}$ to be ordered with time increasing from left to right. The projections correspond to propositions about the system in the usual way. For example, a projection onto the $\sigma_{z}=1 / 2$ eigenspace of a spin- $1 / 2$ particle applied at time $t$ corresponds to the statement that the particle was in the $\sigma_{z}=1 / 2$ eigenstate at the relevant time. The consistent histories formulation differs from Copenhagen quantum mechanics, however, in that there is no dynamical projection postulate attached to statements of this type.

Suppose now we have a list of sets $\sigma_{j}$ of this form. Then the histories given by choosing one projection from each $\sigma_{j}$ in all possible ways are an exhaustive and exclusive set of alternatives. We use Gell-Mann and Hartle's decoherence condition, and say that $\mathcal{S}$ is a consistent set of histories if

$$
\operatorname{Tr}\left(P_{n}^{i_{n}} \ldots P_{1}^{i_{1}} \rho P_{1}^{j_{1}} \ldots P_{n}^{j_{n}}\right)=\delta_{i_{1} j_{1}} \ldots \delta_{i_{n} j_{n}} p\left(i_{1} \ldots i_{n}\right)
$$

or, in the time-neutral case,

$$
\operatorname{Tr}\left(\rho_{f} P_{n}^{i_{n}} \ldots P_{1}^{i_{1}} \rho_{i} P_{1}^{j_{1}} \ldots P_{n}^{j_{n}}\right)=\delta_{i_{1} j_{1}} \ldots \delta_{i_{n} j_{n}} p\left(i_{1} \ldots i_{n}\right)
$$

When $\mathcal{S}$ is consistent, $p\left(i_{1} \ldots i_{n}\right)$ is the probability of the history $\left\{P_{1}^{i_{1}}, \ldots, P_{n}^{i_{n}}\right\}$. We say the set

$$
\mathcal{S}^{\prime}=\left\{\sigma_{1}, \ldots, \sigma_{k}, \tau, \sigma_{k+1}, \ldots, \sigma_{n}\right\}
$$

is a consistent extension of a consistent set of histories $\mathcal{S}=\left\{\sigma_{1}, \ldots, \sigma_{n}\right\}$ by the set of projections $\tau=\left\{Q^{i}: i=1, \ldots, m\right\}$ if $\tau$ is a projective decomposition and $\mathcal{S}^{\prime}$ is consistent.

Suppose now that we have a collection of data defined by the history

$$
H=\left\{P_{1}^{i_{1}}, \ldots, P_{n}^{i_{n}}\right\}
$$

which has non-zero probability and belongs to the consistent set $\mathcal{S}$. This history might, for example, describe the results of a series of experiments or the observations made by an observer. To make scientific use of the formalism we then want to make further inferences from the data. In the standard formalism, this can only be done relative to a choice of consistent extension $\mathcal{S}^{\prime}$ of $\mathcal{S}$.2 Once $\mathcal{S}^{\prime}$ is fixed we can make probabilistic inferences

2 We consider only the standard formalism here. It is possible to amend the formalism by appending axioms which identify natural retrodictions [3, 12, 13. If only these retrodictions are allowed, contrary retrodictions are avoided. However, this would also exclude almost all scientifically desirable retrodictions 13 . 
conditioned on the history $H$. For example, if $\mathcal{S}^{\prime}$ has the above form, the histories extending $H$ in $\mathcal{S}^{\prime}$ are $H^{i}=\left\{P_{1}^{i_{1}}, \ldots, P_{k}^{i_{k}}, Q^{i}, P_{k+1}^{i_{k+1}}, \ldots, P_{n}^{i_{n}}\right\}$ and the history $H^{i}$ has conditional probability $p\left(H^{i}\right) / p(H)$.

We use the convention that the calculation is made at the time of the last event from the history, so that any projection occurring before this last event is a retrodiction. Thus if $k=n$ then $p\left(H^{i}\right) / p(H)$ is the probability with which the proposition corresponding to the projection $Q^{i}$ is predicted; if $k<n$ it is the probability with which the proposition is retrodicted. The different $\mathcal{S}^{\prime}$ are to be thought of as different, equally valid, possible pictures of the past and future physics of the system, or more formally as different and generally incompatible logical structures allowing different classes of inferences from the given data.

\section{Contrary retrodictions and predictions}

We now give two simple examples of contrary inference in the consistent histories formalism. The Hilbert space $\mathcal{H}$ is taken to be of dimension greater than or equal to three.

Example $1 \quad$ Take $\rho=|a\rangle\langle a|$, where the normalised vector $|a\rangle$ defines the initial state of the system. Define the projection $P_{c}=|c\rangle\langle c|$, for some normalised vector $c$ such that $0<|\langle a \mid c\rangle| \leq 1 / 3$. Suppose that the data correspond to the history $\mathcal{H}=\left\{P_{c}\right\}$ from the consistent set $\mathcal{S}=\left\{\left\{P_{c}, 1-P_{c}\right\}\right\}$. Now consider a consistent extension of the form $\mathcal{S}^{\prime}=\left\{\left\{P_{b}, 1-P_{b}\right\},\left\{P_{c}, 1-P_{c}\right\}\right\}$, where $P_{b}=|b\rangle\langle b|/|\langle b \mid b\rangle|$ for some unnormalised vector $|b\rangle$ with the property that

$$
\langle c \mid b\rangle\langle b \mid a\rangle=\langle c \mid a\rangle\langle b \mid b\rangle
$$

It is not hard to verify that $\mathcal{S}^{\prime}$ is consistent and that the conditional probability of $P_{b}$ given $H$ is 1 . It is also easy to see that there are at least two mutually orthogonal vectors $|b\rangle$ satisfying (3.1). For example, let $\left|v_{1}\right\rangle,\left|v_{2}\right\rangle,\left|v_{3}\right\rangle$ be orthonormal vectors and take $|a\rangle=\left|v_{1}\right\rangle$ and $|c\rangle=\lambda\left|v_{1}\right\rangle+\mu\left|v_{2}\right\rangle$, where $\lambda^{2}+\mu^{2}=1$ and we may take $\lambda$ and $\mu$ real. Then the vectors

$$
\left|b_{ \pm}\right\rangle=\lambda\left|v_{1}\right\rangle+\frac{\mu}{x}\left|v_{2}\right\rangle \pm \frac{(x-1)^{1 / 2} \mu}{x}\left|v_{3}\right\rangle
$$

both satisfy (3.1) and are orthogonal if $x$ is real and $x^{2} \lambda^{2}=(x-2)\left(1-\lambda^{2}\right)$, which has solutions for $\lambda \leq 1 / 3$. Thus this construction produces consistent sets which give contrary probability one retrodictions. 
Example 2 Now consider the formalism applied to generalised quantum mechanics, choose vectors $|a\rangle,|b\rangle$ and $|c\rangle$ as above, take $\rho_{i}=|a\rangle\langle a|$ and $\rho_{f}=P_{c} / C$, where the normalisation constant $C=|\langle a \mid c\rangle|^{2}$. Suppose that the data correspond to the history $\mathcal{H}=\left\{P_{a}\right\}$ from the consistent set $\mathcal{S}=\left\{\left\{P_{a}, 1-P_{a}\right\}\right\}$, and consider consistent extensions of the form $\mathcal{S}^{\prime}=\left\{\left\{P_{a}, 1-P_{a}\right\},\left\{P_{b}, 1-P_{b}\right\}\right\}$ where $P_{b}$ is as in Example 1. As above, the conditional probability of $P_{b}$ given $H$ is 1 , so that we obtain consistent sets which give mutually contrary probability one predictions.

Note that it is impossible to produce an example in which the formalism makes contrary predictions when applied to ordinary quantum mechanics. In this context, if $P$ is predicted with probability 1 from the history $H=\left\{P_{1}, \ldots, P_{n}\right\}$ in the set $\mathcal{S}$, then

$$
P P_{n} \ldots P_{1} \rho^{1 / 2}=P_{n} \ldots P_{1} \rho^{1 / 2}
$$

and

$$
Q P_{n} \ldots P_{1} \rho^{1 / 2}=0
$$

if $Q \leq(1-P)$. Thus if a projection $Q$ orthogonal to $P$ belongs to any consistent set then its probability in that set, conditional on the history $H$, is zero. It is also easy to see that we can construct examples in which any number of consistent sets make mutually contrary retrodictions - or, in the case of time-neutral quantum mechanics, predictions - by taking the dimension of $\mathcal{H}$ to be sufficiently large and choosing $|\langle a \mid c\rangle|$ sufficiently small.

Though the contrary inferences in the above example both correspond to onedimensional projections, it is easy to construct similar examples of contrary inferences corresponding to projections of different dimension since, given the above initial and final states, the condition for a projection $P$ to correspond to a probability one consistent inference is simply that $\langle a|P| c\rangle=\langle a \mid c\rangle$. Thus contrary inferences could not be avoided by introducing a unitary equivalence relation — perhaps along the lines of those recently considered by Gell-Mann and Hartle[14] - according to which any pairs of projections involved in contrary inferences are declared physically equivalent. 


\section{Conclusions}

The incompatibility of the logics corresponding to different consistent sets is generally described as a natural generalisation of the principle of complementarity in Copenhagen quantum mechanics: a discussion making precisely this point can be found, for example, in Chapter 5.4 of Omnès' recent book 4 . There is, though, no parallel in standard quantum mechanics for the prediction and retrodiction of contrary propositions, and many might feel that no acceptable interpretation of quantum theory should allow such inferences. Indeed, Omnès comments that "The worst event would be if two different ways of reasoning could lead to different conclusions when one is using two different consistent logics. In view of this danger, which would mean that the present approach is completely wrong, we shall initially discuss how two different logics can be related to each other." [15]

Now Omnès has in mind here a slightly different possibility, namely that if two propositions both belong to two distinct consistent sets, and one implies the other in one set, the implication might fail in the other set. This cannot happen in the consistent histories formalism. It is not possible, for example, to use the same set of data to predict the proposition $P$ in one set and its negation $(1-P)$ in another, both with probability one. At first sight it may seem as though the above examples do precisely this. The reason why they fail to do so is that, in the consistent histories formalism, if we have two propositions corresponding to projection operators $P \leq Q$ (i.e. the range of $P$ is a subspace of that of $Q)$ and if $P$ is predicted with probability one, it does not follow that $Q$ is predicted with probability one (or with any other probability).

It might possibly be argued that this last feature is less of a flaw, and that the examples above are less worrying, than the type of contradictory inference Omnès considers - but it is hard to see why. The fact that the theory stipulates that the pictures corresponding to different sets are incompatible alternatives cannot be used as a defence here without allowing the same defence in the case of Omnès' hypothetical disaster. Clearly, no logical contradiction arises if we suppose that different consistent sets simply give different pictures of the physics and if we make no stipulation whatsoever about the relationship between these pictures. But the same of course is true of the analogous supposition about inconsistent sets of histories. To justify the fundamental assumption of the consistent histories formalism - that it is precisely the consistent sets which give sensible physical descriptions - we need to suppose the following. First, that it is wrong - a product of misguided classical intuition - to suppose that contrary propositions $P$ and $Q$ should 
never be inferred with probability one in different sets. Second, that it is right - a fundamental feature of quantum physics - to suppose that contradictory propositions $P$ and $(1-P)$ should never be inferred in different sets. This is certainly not the standard understanding of the situation, and there seems no obvious reason to adopt it. One might plausibly try to argue that both suppositions are right, or conceivably (depending on how the descriptions are to be used) that both are wrong, but it seems particularly hard to argue for one and against the other.

Another possible counter-argument is that, in the end, scientists need only worry about predictions, and contrary predictions can be avoided by restricting the formalism to standard, rather than generalised, quantum mechanics. One difficulty with this line of defence is that it is the retrodictive cosmological applications of the consistent histories formalism that are presently the most interesting. Unlike other approaches to quantum theory, the formalism allows us to discuss series of past cosmological events and to assign probabilities to them, even when some or all of the events occurred before the formation of classical structures. Prediction, on the other hand, is where the consistent histories formalism is at its weakest. No coherent interpretation of the formalism has been found which unambiguously implies the standard predictions of Copenhagen quantum mechanics, although those predictions (among many others) can be reproduced by calculations within the formalism. Moreover, though the formalism allows many different predictive calculations, those which are new seem to be physically irrelevant except in highly implausible scenarios and, possibly, in the case of generalised time-neutral quantum cosmology. This, though, is precisely the case in which contrary predictions arise.

If we reject these defences we seem to be left with the conclusion that the contrary inferences implied by the consistent histories formalism make it hard to take it seriously as a fundamental theory in its present form. This means that further constraints beyond consistency are needed in order to construct a natural generalisation of the Copenhagen interpretation to closed systems. Whether physically sensible and mathematically precise constraints can be found in standard versions of the formalism, such as the one above, is an important and intriguing open question. It might also be interesting to investigate the analogous problem in the more abstract schemes characterising the logical structure of consistent histories which have recently been developed. [16] 


\section{Note added}

After writing this Letter, my attention was drawn to Cohen's interesting analysis [17] of an example due to Aharonov and Vaidman[18]. Ref. [17] includes what seems to be the

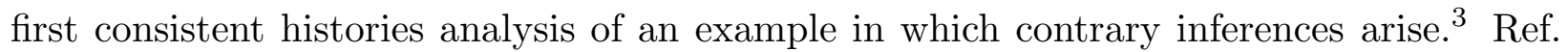
[17] is a critique of Aharonov and Vaidman's arguments rather than those of the consistent histories literature, so that the implications for the consistent histories formalism are not considered in any detail.

A response to the present Letter, defending the consistent histories formalism, can be found in Ref. [19]. Griffiths stresses the point, emphasized above, that the consistent histories formalism can be interpreted in a way which leads to no logical contradiction.

As mentioned above, it seems that further natural constraints beyond consistency seem to be needed for a sensible formulation of the quantum theory of closed systems. It turns out that at least one such constraint exists: a stronger version of the consistent histories formalism, designed to avoid the problems discussed in this paper, is described in Ref. [20.

\section{Acknowledgements}

I am very grateful to Oliver Cohen, Fay Dowker, Arthur Fine and Lucien Hardy for helpful discussions and to Bob Griffiths and Jim Hartle for critical readings of the manuscript and valuable comments. I thank the Royal Society for financial support.

3 The argument of the relevant section, VIII B, of Ref. [17] is not entirely correct: the statement that failure of consistency follows from the failure of the relevant projection operators to commute is false. 


\section{References}

[1] R.B. Griffiths, J. Stat. Phys. 36 (1984) 219.

[2] R.B. Griffiths, Found. Phys. 23 (1993) 1601.

[3] R. Omnès, J. Stat. Phys. 53 (1988) 893.

[4] R. Omnès, The Interpretation of Quantum Mechanics, Princeton University Press, Princeton (1994).

[5] M. Gell-Mann and J.B. Hartle in Complexity, Entropy, and the Physics of Information, SFI Studies in the Sciences of Complexity, Vol. VIII, W. Zurek (ed.), Addison Wesley, Reading (1990).

[6] M. Gell-Mann and J.B. Hartle in Proceedings of the NATO Workshop on the Physical Origins of Time Asymmetry, Mazagón, Spain, September 30-October 4, 1991, J. Halliwell, J. Pérez-Mercader, and W. Zurek (eds.), Cambridge University Press, Cambridge (1994); gr-qc/9304023.

[7] M. Gell-Mann and J.B. Hartle, Phys. Rev. D 47 (1993) 3345.

[8] F. Dowker and A. Kent, J. Stat. Phys. 82 (1996) 1575.

[9] A. Kent, Phys. Rev. A 54 (1996) 4670.

[10] F. Dowker and A. Kent, Phys. Rev. Lett. 75 (1995) 3038.

[11] Y. Aharonov, P. Bergmann and J. Lebowitz, Phys. Rev. B 134 (1964) 1410.

[12] R. Omnès, Found. Phys. 25 (1995) 605.

[13] F. Dowker and A. Kent, unpublished.

[14] M. Gell-Mann and J.B. Hartle, gr-qc/9404013 (unpublished).

[15] Page 161 of 团.

[16] C.J. Isham, J. Math. Phys. 23 (1994) 2157; C.J. Isham and N. Linden, J. Math. Phys. 35 (1994) 5452.

[17] O. Cohen, Phys. Rev. A 51 (1995) 4373.

[18] Y. Aharonov and L. Vaidman, J. Phys. A 24 (1991) 2315.

[19] R.B. Griffiths, Phys. Rev. A 54 (1996) 2759.

[20] A. Kent, "Quantum Histories and Their Implications", gr-qc/9607073, submitted to Ann. Phys. 